\title{
Dual Hollow-Core Anti-Resonant Fibres
}

\author{
N.V. Wheeler*, T.D. Bradley, J.R. Hayes, G.T. Jasion, Y. Chen, S.R. Sandoghchi, P. Horak, \\ F. Poletti, M.N. Petrovich and D.J. Richardson. \\ Optoelectronics Research Centre, University of Southampton, Highfield Campus, SO17 1BJ, \\ Southampton, UK \\ *n.v.wheeler@soton.ac.uk
}

\begin{abstract}
While hollow core-photonic crystal fibres are now a well-established fibre technology, the majority of work on these speciality fibres has been on designs with a single core for optical guidance. In this paper we present the first dual hollow-core anti-resonant fibres (DHC-ARFs). The fibres have high structural uniformity and low loss (minimum loss of $0.5 \mathrm{~dB} / \mathrm{m}$ in the low loss guidance window) and demonstrate regimes of both inter-core coupling and zero coupling, dependent on the wavelength of operation, input polarisation, core separation and bend radius. In a DHC-ARF with a core separation of $4.3 \mu \mathrm{m}$, we find that with an optimised input polarisation up to $65 \%$ of the light guided in the launch core can be coupled into the second core, opening up applications in power delivery, gas sensing and quantum optics.
\end{abstract}

Keywords: Hollow core photonic crystal fibres, microstructured fibres, high power delivery, gas sensing, fibre fabrication.

\section{INTRODUCTION}

Hollow core-photonic crystal fibres (HC-PCFs) provide low loss optical guidance in an air core surrounded by a periodic array of up to several hundred air holes. In recent years rapid progress in the design and fabrication of $\mathrm{HC}^{-\mathrm{PCF}}{ }^{1}$ has been reported. Two distinct families of HC-PCFs have emerged which are distinguished by their guidance mechanism: hollow core-photonic bandgap fibres (HC-PBGFs) and hollow core anti-resonant fibres (HC-ARFs).

While both fibre groups provide the main benefits associated with HC-PCFs (e.g., ultimate low latency and ultra-low non-linearity ${ }^{2}$ ), HC-PBGFs typically provide lower attenuation over a narrower spectral window (e.g., up to $200 \mathrm{~nm}$ in the $1550 \mathrm{~nm}$ region $\left.{ }^{3}\right)$ in a hollow core with a smaller diameter $(\sim 25 \mu \mathrm{m}$ at $1550 \mathrm{~nm})$ and as such are suited to applications in telecommunications ${ }^{3}$ and nonlinear optics ${ }^{4}$. On the other hand, the anti-resonant guidance mechanism enables greater freedom in the fibre design as the core wall thickness and shape are the critical parameters in defining the fibre properties while the cladding geometry plays only a relatively minor role. This freedom opens up the potential for simpler fibre designs and large core diameters (up to $\sim 100 \mu \mathrm{m}^{5}$ ), which provide low loss guidance over broad spectral windows and makes HC-ARFs suitable for applications such as high power delivery and gas and vapour based non-linear optics. Recent advances in the understanding of the core wall geometry in HC-ARFs and its impact on fibre attenuation ${ }^{6,7}$ have also enabled HC-ARFs to begin to compete with HC-PBGFs in terms of fibre attenuation at short wavelengths $(\leq \sim 1 \mu \mathrm{m})$ and in the mid-IR ${ }^{8}$.

The vast majority of work related to HC-PCFs and in silica core PCFs has been focussed on geometries with a single core defect within the cladding structure. However, there have been several theoretical and experimental reports of dual (solid) core PCFs with coupled cores ${ }^{9-15}$. In particular the characteristics of dual solid core PBGFs have been studied and interesting properties such as decoupling of the cores at the shorter wavelength edge of the bandgap and inversion of the usual order of the super-modes with respect to propagation constant were found ${ }^{12}$. Recently, a new multicore HC-PBGF (known as a PRISM fibre) was demonstrated ${ }^{16}$ with a central core for low loss guidance surrounded by smaller 'shunt' cores which remove higher order modes from the central core through inter-core coupling to enable effectively single mode guidance in the primary core; these fibres show how multi-hollow core structures have potentially interesting and unique optical properties which cannot otherwise be achieved. A key challenge in achieving strong coupling between two modes in separate optical waveguides is that the geometry of the waveguides must be extremely uniform and similar 
in structure, requiring strict fabrication tolerances; in a PRISM fibre small geometry differences can be overcome by coiling the fibre to encourage the necessary phase matching.

Due to the simpler cladding geometry and greater freedom in fibre design offered by HC-ARFs as compared to HCPBGFs, a more straightforward fabrication process can be used which makes it easier to meet the necessary fabrication tolerances to ensure strong coupling between the separate hollow cores and therefore to enable access to a wider range of applications. However, there has only been one study on dual core HC-ARF previously reported ${ }^{17}$, fabricated using a polymer matrix, where fibres with a $4 \mu \mathrm{m}$ gap between the cores and a single strut between the cores were reported. In that study, no inter-core coupling was recorded in the anti-resonant transmission band for either fibre ${ }^{17}$. This lack of coupling was attributed to both the high modal confinement provided in HC-ARFs and also structural differences between the two cores which led to a significant difference between the propagation constants of the modes guided in each core.

Here we report, for the first time, dual core silica HC-ARFs (DHC-ARFs). We show that these fibres have low loss ( $\sim .5$ - $3 \mathrm{~dB} / \mathrm{m}$ across a wide transmission window) and demonstrate regimes of both inter-core coupling and zero coupling, dependent on guidance wavelength, input polarization, core separation and bend radius. In section 2 , we describe the fabrication process and key fibre properties. In section 3, the fibre characterisation and power transfer between the two cores are discussed and we conclude in section 4.

\section{FIBRE FABRICATION}

The DHC-ARFs were fabricated using the stack and draw method; each core was introduced by removing one element in the stacked preform. The fibres were designed to have a cladding structure with a Kagomé lattice. As mentioned in the previous section, the use of this HC-ARF design enables much more freedom as compared to a HC-PBGF design. In the initial stacking process, significantly fewer capillaries are required due to the reduced number of cladding rings needed for low loss optical guidance (strictly only one ring is required for anti-resonant guidance and further rings can have benefits in terms of bend robustness in the final fibre ${ }^{18}$ ). Furthermore, with the anti-resonant guidance mechanism, a core defect can be formed by removing just a single element from the lattice, as opposed to removing a minimum of 3 elements for a HC-PBGF. Finally with a HC-ARF design there is much more freedom in the parameters of the final fibre in terms of the cladding pitch, core diameter and outer diameter of the fibre. In this work we aimed to fabricate DHCARFs demonstrating strong inter-core coupling and therefore care was taken in both draw stages to keep the core geometries uniform.

One of our initial fibres is shown in Fig. 1(a); it has two 1-cell core elements surrounded by three cladding rings which are separated by a single smaller capillary. The maximum core diameters for the two cores are $44.9 \mu \mathrm{m}$ and $45.3 \mu \mathrm{m}$ respectively, the core wall thickness varies between $\sim 400$ and $500 \mathrm{~nm}$ and the central gap between the two cores is 10.0 $\mu \mathrm{m}$ (Fig. 1(b)). Although the optical properties of these fibres are complex and require bespoke modelling tools to predict, a simple picture based on mode coupling theory predicts that the fibre length over which strong inter-core

(a)

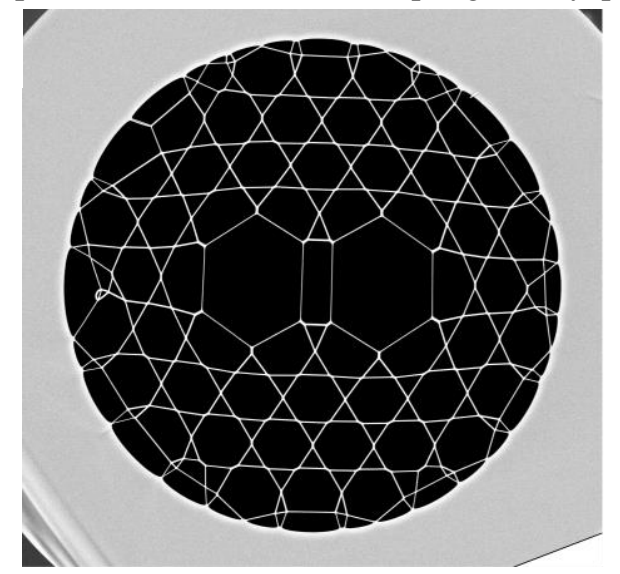

(b)

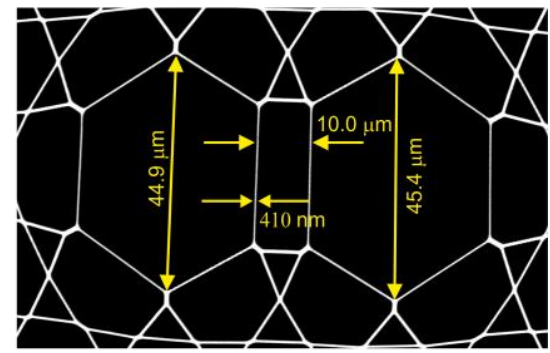

Figure 1: (a) Scanning electron microscope image of a DHC-ARF (b) Detail of the dual core geometry. 
coupling will be observed will be strongly defined by the core separation. We therefore used differential pressurization of the hole separating the two cores in order to obtain fibres with widely different inter-core separation and thus produce a systematic study of the fibre properties as a function of this parameter.

\section{FIBRE CHARACTERISATION}

Several fibres were fabricated with different core separations in order to investigate the parameters which control the power transfer between the two cores. Three examples with core separations of $9.7 \mu \mathrm{m}, 7.5 \mu \mathrm{m}$ and $4.3 \mu \mathrm{m}$ are shown in Figure 2(a)-(c) and are referred to as Fibres A, B and C respectively. These fibres were optically measured using two techniques to characterize their transmission properties and the inter-core coupling.

(a)

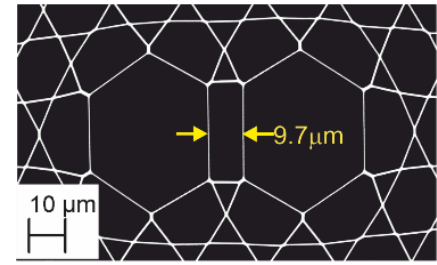

(b)

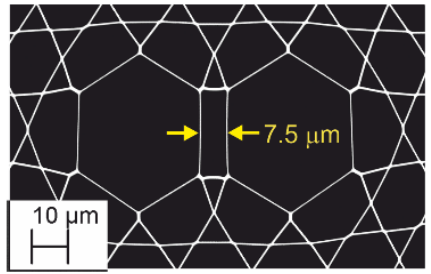

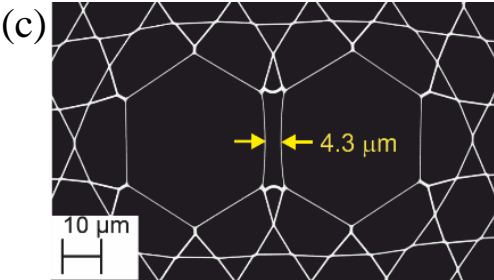

Figure 2(a)-(c): Scanning electron micrograph images of Fibres A - C.

\subsection{White Light Source Characterization}

In the first technique, an incandescent white light source (WLS) was coupled into a short length of LMA-25 (NKT Photonics) which was used as a launch fibre into the DHC-ARF as it has a similar mode field diameter to that expected for each individual core. At the input to the DHC-ARF, light was selectively launched into only one core; the core selection was confirmed by using a silicon camera (operating for wavelengths below $1.1 \mu \mathrm{m}$ ) to image the fibre output. At the short wavelengths at which this camera operates no inter-core coupling was observed and therefore it was clear that the light was successfully launched into one core only. Following this initial alignment, the light at the output of the DHC-ARF was collected from each core individually, again using LMA-25, and coupled into an optical spectrum analyser (OSA). This technique enabled observation of the inter-core coupling over the broadband transmission window of the fibre.

Figures 3(a) and (b) show the results of this measurement for a 1 metre long sample of fibre $\mathrm{C}$ for light launched into core 1 and core 2 respectively, recorded with a resolution of $5 \mathrm{~nm}$. The loss of fibre $\mathrm{C}$ was also measured using this set up but with the DHC-ARF directly coupled into the OSA and the results are shown in Fig. 3(c). In the lowest loss spectral region $(\sim 1.1-1.7 \mu \mathrm{m})$ the attenuation varies from $\sim 1-3 \mathrm{~dB} / \mathrm{m}$. Similar behaviour was observed for fibres A and $\mathrm{B}$, with a minimum loss of $\sim 0.5 \mathrm{~dB} / \mathrm{m}$. Figures 3 (a) and (b) clearly show that light is coupled from core 1 to core 2 and that this coupling is strongly wavelength dependent; at wavelengths below $1200 \mathrm{~nm}$ negligible coupling occurs between the two cores and the light stays confined in the launch core. At wavelengths beyond $1200 \mathrm{~nm}$, inter-core coupling is recorded and the strongest coupling occurs between 1350 and $1500 \mathrm{~nm}$. All of the measured traces are highly structured; if each core supported only single mode guidance and the fibre transmission loss was spectrally flat then we would expect to see intensity variations with wavelength that correspond to just the power transfer between the two cores. However, from Fig. 3(a) and (b) it is clear that the intensity variations are highly complex; two key factors which contribute to this are firstly, that these fibres are not single mode (see section 3.2) and also that the transmission spectra of this type of HC-ARF are typically punctuated by loss peaks due to resonances between the core guided modes and nodes in the core surround and, to a lesser extent, the cladding structure. This technique, while enabling broadband analysis, is clearly limited in terms of resolution and due to this complex variation of intensity with wavelength does not reveal the detail in the power transfer between the two cores. 
(a)

(c)

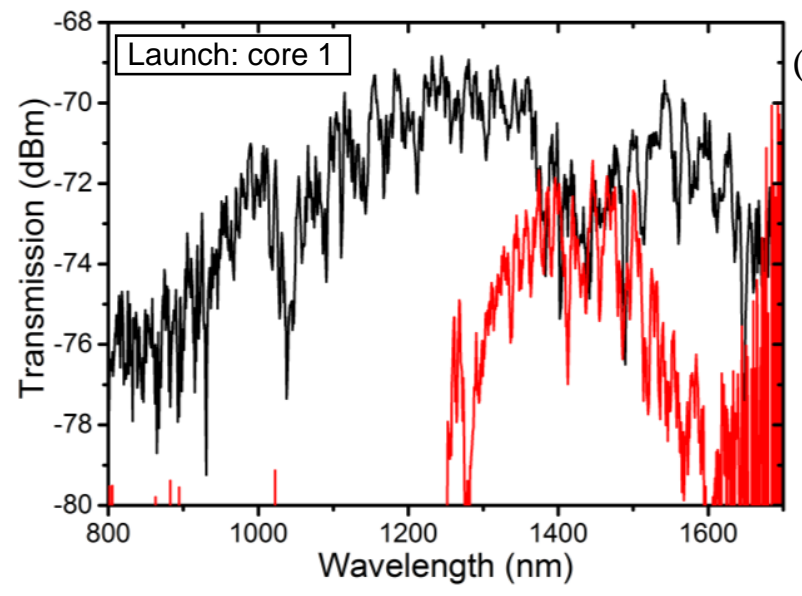

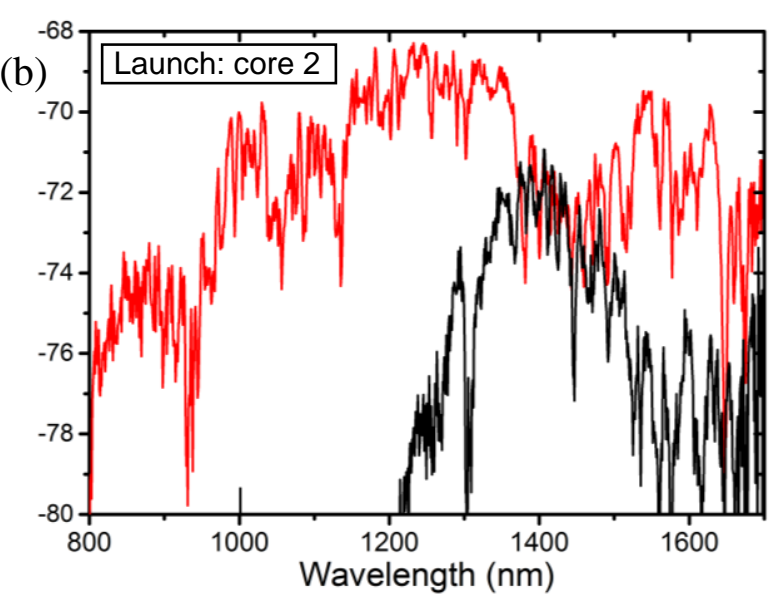

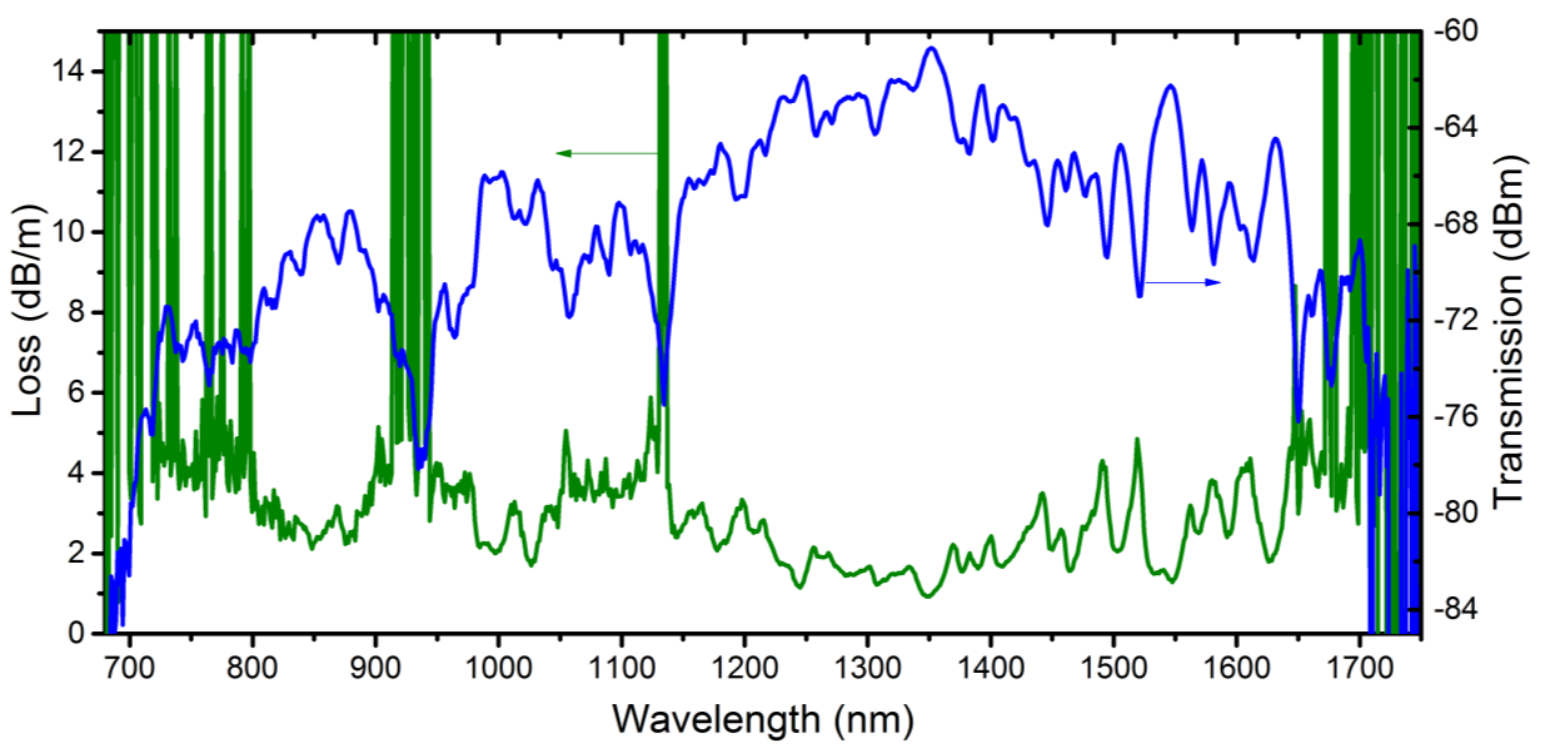

Figure 3: (a) and (b) Transmission spectra recorded at the output of $1 \mathrm{~m}$ in each core of fibre $\mathrm{C}$ for light launched into core 1 (black (black line) and core 2 (red line) respectively., (c) $2.8 \mathrm{~m}$ transmission spectrum (blue line) and cutback loss measurement (green line) for fibre $\mathrm{C}$ (from $5 \mathrm{~m}$ to $2.8 \mathrm{~m}$ ).

\subsection{Tuneable Laser Source Characterization}

In order to measure further the power transfer between the two hollow cores a second characterisation approach was used (Fig. 4). A tuneable laser source (TLS) was aligned into one core at the fibre input (via a SMF, polarisation optics and a lens system to match the mode field diameter of each individual core) and the image at the fibre output was captured by a CCD camera as the TLS was scanned from 1520 to $1630 \mathrm{~nm}$ (10 pm step-size). This technique provides high spectral resolution, a measurement of the output mode intensity profile and also polarisation control at the input to the DHC-
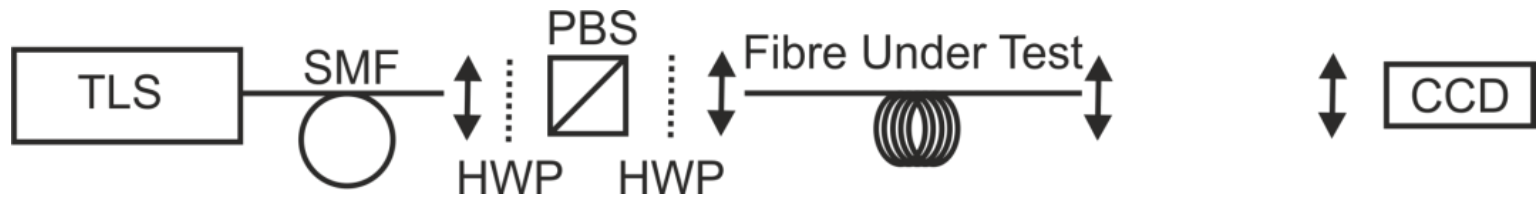

Figure 4: Experimental set-up for measuring the power transfer between the two cores in the DHC-ARF using a tuneable laser source (TLS) coupled via a single mode fibre (SMF), lens arrangement and polarization control (HWP: half wave plate, PBS: polarizing beam splitter) into the fibre under test (DHC-ARF). The output of the fibre under test is recorded on a CCD camera. 
ARF. The CCD images were analysed to find the power in each core as a function of wavelength. This technique was applied to $2 \mathrm{~m}$ lengths of each of the fibres A-C. For each set of results the polarisation at the input to the DHC-ARF was optimised to maximise the power transfer between the cores.

Using this technique on fibre $\mathrm{A}$, which has a core separation of $9.7 \mu \mathrm{m}$, produced a maximum power transfer of $\sim 10 \%$ (normalised to the total power) from the launch core to the second core. Figure 5(a) and (b) compare the power in each core as a function of wavelength for fibres B and C. These fibres have a core separation of 7.5 and $4.3 \mu \mathrm{m}$ and a maximum power transfer of $\sim 50$ and $\sim 65 \%$ respectively, clearly indicating an increase in coupling strength as the core separation is decreased. Interestingly, in a further fibre where the cores were not separated by a hole but instead by a short strut contact point, negligible coupling was observed; further characterisation to understand this is underway.

We have also observed that the coupling between the two cores is strongly polarization-dependent. The plots in Fig 5(a) and (b) were obtained by aligning the polarization at input in such a way that the coupling was maximized. We found that, by rotating the input polarization by 30 degrees from this condition, inter-core coupling was greatly suppressed as shown in Fig. 5(c) for Fibre C. We speculate that this is related to the hexagonal symmetry of the fibre but further
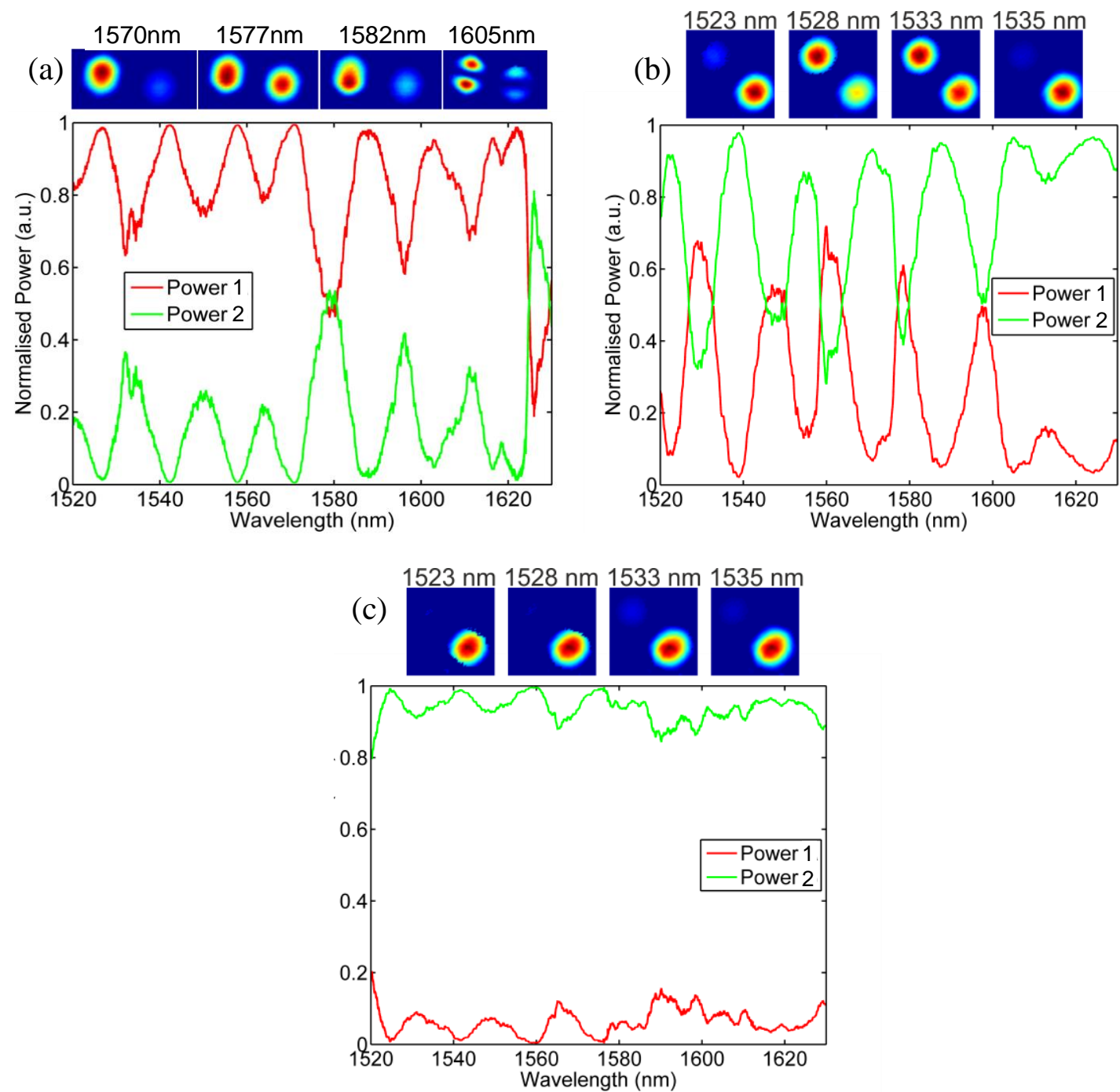

Figure 5: (a) Experimental results showing the power in cores 1 and 2 (at the output of a 2 metre length) of Fibre B as a function of wavelength, normalized to the total power transmitted in the fibre, (b) and (c) same as (a) but measured using Fibre C, for optimized and non-optimized input polarization respectively. 
analysis is underway. This polarization dependent coupling behaviour was the same for both cores. Furthermore there is evidence that the cores also support higher order modes (in particular the LP L $_{11}$ mode) which can be excited and transmitted over the lengths analysed here, and can also be coupled between the two cores. This is clearly shown in the inset to Figure 5(a) at $1605 \mathrm{~nm}$. The fibre loss at this wavelength indicates that this corresponds to a spectral region where the fundamental mode is not guided with low loss.

These results on the coupling between the two hollow cores in DHC-ARF confirm that strong inter-core coupling can be achieved through careful control of the fibre structure during fabrication. We believe that with further optimisation even stronger coupling can be achieved (i.e. further reduction of the gap between the cores while avoiding contact and improved core symmetry). These results are in contrast to the only previous study on DHC-ARF ${ }^{17}$ where no coupling between the two cores was recorded and this was partly attributed to the high degree of confinement in a HC-ARF; here we have clearly demonstrated that this high degree of confinement in the hollow core is not a barrier to inter-core coupling.

Initial tests have also been carried out to determine the sensitivity of the coupling to bending; preliminary results show that at moderate bend radius $(\sim 10 \mathrm{~cm})$ the core transmission starts to suffer from bend loss and also that the coupling length is affected by bend. These studies are ongoing to determine how robust and repeatable this coupling sensitivity is. Furthermore, very early tests have shown that in the strong coupling regime the inter-core coupling is highly sensitive to vibration and it will scientifically interesting to investigate more in detail the potential of these fibres in the sensor application workspace. In addition to applications in sensing these fibres hold promise for devices such as couplers and splitters as well as in novel gas sensing and quantum optics experiments.

\section{CONCLUSIONS}

We have successfully fabricated the first dual hollow core anti-resonant fibre which demonstrates optical coupling between the two cores. A range of fabricated fibres have been characterised and the highest level of coupling between the two hollow cores was found for a fibre with high structural uniformity and with a core separation of $4.3 \mu \mathrm{m}$. For this fibre we found that, with optimised input polarisation, up to $65 \%$ of the light guided in the launch core can be transferred into the second core showing the anti-resonant guidance mechanism is not prohibitive for achieving strong coupling between neighbouring cores. Initial results have also shown the sensitivity of the inter-core coupling to both bend and vibration, to an extent dependent on fibre design and operating wavelength.

\section{|ACKNOWLEDGEMENTS}

N.V. Wheeler gratefully acknowledges support from a Royal Society University Research Fellowship.

\section{REFERENCES}

[1] Poletti, F., Petrovich, M.N. and Richardson, D.J., "Hollow-core photonic bandgap fibers: technology and applications," Nanophotonics 2(5-6), 315-340 (2013).

[2] Poletti, F., Wheeler, N.V., Petrovich, M.N., Baddela, N., Numkam Fokoua, E., Hayes, J.R., Gray, D.R., Li, Z., Slavik, R. and Richardson, D.J., "Towards high-capacity fibre optic communications at the speed of light in a vacuum," Nature Photonics 7, 279-284 (2013).

[3] Chen, Y., Liu, Z., Sandoghchi, S., Jasion, G., Bradley, T., Numkam Fokoua, E., Hayes, J., Wheeler, N.V., Gray, D., Mangan, B., Slavík, R., Poletti, F., Petrovich, M.N. and Richardson, D.J., "Multi-kilometer long, longitudinally uniform Hollow Core Photonic Bandgap Fibers for broadband low latency data transmission," Journal of Lightwave Technology, 34(4) 1-10 (2016).

[4] Gérôme, F., Dupriez, P., Clowes, J., Knight, J. C. and Wadsworth, W.J., "High power tunable femtosecond soliton source using hollow-core photonic|bandgap fiber, and its use for frequency doubling," Optics Express 16(4) 23812386 (2008).

[5] Cheng, Y., Wang, Y.Y. and Benabid, F., "Fabrication and characterization of ultra-large core size $(>100 \mu \mathrm{m})$ Kagome fibre for laser power handling," in CLEO 2011 CTuE1 (2011). 
[6] Wang, Y.Y., Wheeler, N.V., Couny, F., Roberts, P. J. and Benabid, F., "Low loss broadband transmission in hypocycloid-core Kagome hollow-core photonic crystal fiber," Optics Letters 36(5) 669-671 (2011).

[7] Debord, B., Alharbi, M., Bradley, T., Fourcade-Dutin, C., Wang, Y.Y., Vincetti, L., Gérôme, F. and Benabid, F. "Hypocycloid-shaped hollow-core photonic crystal fiber Part I: Arc curvature effect on confinement loss," Optics Express 21(23) 28597-28608 (2013).

[8] Yu, F. and Knight, J.C., "Spectral attenuation limits of silica hollow core negative curvature fiber," Optics Express 21(18) 21466- 21471 (2013).

[9] Mangan, B.J., Knight, J.C., Birks, T.A., Russell, P.St.J. and Greenaway, A.H., "Experimental study of dual-core photonic crystal fibre," Electronics Letters 36(16) 1358-1359 (2000).

[10] Saitoh, K., Sato, Y.and Koshiba, M., "Coupling characteristics of dual-core photonic crystal fiber couplers," Optics Express 11(24) 3188-3195 (2003).

[11] Wang, Z., Kai, G., Liu, J., Zhang, C., Sun, T., Wang, C., Zhang, W., Yuan, S. and Dong, X., "Coupling and decoupling of dual-core photonic bandgap fibers," Optics Letters 30(19) 2542-2544 (2005).

[12] Wang, Z., Taru, T., Birks, T.A. and Knight, J.C., "Coupling in dual-core photonic crystal fibers: theory and experiment," Optics Express 15(8) 4795-4803 (2007).

[13] Padden, W.E.P., van Eijkelenborg, M.A., Argyros, A. and Issa, N.A., "Coupling in a twin-core microstructured polymer optical fiber," Appl. Phys. Lett. 84(10), 1689- 1691 (2004).

[14] Bellanca, G., Riesen, N., Argyros, A., Leon-Saval, S.G., Lwin, R., Parini, A., Love, J.D. and Bassi, P., "Holey fiber mode-selective couplers," Optics Express 23(15) 18888-18896 (2015).

[15]Lian, Z., Horak, P., Feng, X., Xiao, L., Frampton, K., White, N., Tucknott, J.A., Rutt, H., Payne, D.N., Stewart, W. and Loh, W.H., "Nanomechanical optical fiber," Optics Express 20(28) 29386- 29394 (2012).

[16] Fini, J.M., Nicholson, J.W., Mangan, B.M., Meng, L., Winderler, R.S., Monberg, E.M., DeSantolo, A., DiMarcello, F.V. and Mukasa, K., "Polarization maintaining single-mode low-loss hollow-core fibres," Nature Communications 5 1-7 (2014).

[17] Argyros, A., Leon-Saval, S.G. and van Eijkelenborg, M.A., "Twin-hollow-core optical fibres," Optics Communications 282 1785-1788 (2009).

[18] Alharbi, M., Bradley, T. Debord, B., Fourcade-Dutin, C., Ghosh, D., Vincetti, L., Gerome, F., and Benabid, F., "Hypocycloid-shaped hollow-core photonic crystal fiber Part II: cladding effect on confinement and bend loss," Optics Express 21(23) 28609- 28616 (2013). 